\title{
Ranging and Three-Dimensional Imaging Using Time-Correlated Single-Photon Counting and Point-by-Point Acquisition
}

\author{
Gerald S. Buller, Member, IEEE, and Andrew M. Wallace
}

(Invited Paper)

\begin{abstract}
Time-correlated single-photon counting techniques have been applied to time-of-flight ranging and imaging. This article describes recent progress in photon-counting systems performing surface mapping using point-by-point acquisition of noncooperative targets at short ranges of the order of 1-50 m, as well as measurements on distributed targets at longer ranges of the order of a $100 \mathrm{~m}$ to a few kilometers. We describe the measurement approach, the signal analysis methodology and algorithm selection.
\end{abstract}

Index Terms-Avalanche photodiodes, distance measurement, imaging, laser radar, Markov processes, maximum likelihood estimation, Monte Carlo methods.

\section{INTRODUCTION}

$\mathbf{T}$ HERE is an increasing need for three-dimensional imaging systems to acquire range and surface profile data for a number of industrial and defense applications [1]. These applications include: surface mapping for metrology and reverse engineering in the aerospace and automotive industries, the scanning of architectural structures, the creation of virtual reality environments for numerous end applications, and low-light level and eye-safe ranging and target identification.

The technique of time-correlated single-photon counting (TCSPC) has been applied to these applications using somewhat different implementations [2]-[5]. In general, a picosecondduration laser pulse is directed toward a noncooperative target and a single-photon detection system is triggered by the scattered optical return. Since each photon return can be regarded as an independent measurement of the photon return time, the collection of multiple returns (typically $>10^{5}$ return events) can yield time measurements with considerably shorter time resolution than the system jitter, which is typically tens of picoseconds. The good depth resolution means that surface profile detail can be measured and that distances between reflecting surfaces can

Manuscript received January 9, 2007; revised June 15, 2007. This work was supported in part by the U.K. Engineering and Physical Sciences Research Council, Swindon, U.K., in part by QinetiQ, U.K., in part by BAE Systems, Farnborough, U.K., in part by Selex, Sutton-on-the-Forest, North Yorkshire, U.K., and in part by the Royal Society, London, U.K.

G. S. Buller is with the Department of Engineering and Physical Sciences, Heriot-Watt University, Riccarton, Edinburgh EH14 4AS, U.K. (e-mail: g.s.buller@hw.ac.uk).

A. M. Wallace is with the Department of Engineering and Physical Sciences, Heriot-Watt University, Riccarton, Edinburgh EH14 4AS, U.K., and also with the University of Edinburgh, Edinburgh EH8 9LE, Scotland, U.K. (e-mail: a.m.wallace@hw.ac.uk).

Digital Object Identifier 10.1109/JSTQE.2007.902850 be resolved. The highly repetitive nature of such an approach is ideal for low scatter surfaces and surfaces at oblique angles. In addition, the use of photon-counting technology can mean that the average power of the output laser can be sufficiently low for eye-safe and low-light level operation, a desirable factor in a number of applications, including those that are defenserelated. Arrayed detection systems, or a scanning approach using an individual single-photon detector, can be employed for full three-dimensional analysis of scenes. This article concentrates on time-of-flight photon-counting systems using individual optimized single-photon avalanche diode detectors, although the basic principles of photon counting have also been used to form three-dimensional depth images using arrays of semiconductor single-photon detectors [4], [5] and single-photon counting microchannel plates with crossed delay lines [3].

In Section II, we describe a prototype photon-counting system designed for very accurate depth measurement at short range, aimed primarily at applications in metrology. In Section III, we describe a system designed for kilometer ranges, with potential for large-scale site measurement and defense applications. In Section IV, we summarize the principal signal processing algorithms that we have applied to the TCSPC data, in general, applicable to both these sensors. We also present an evaluation of the respective system and algorithmic performances, applied to photon-counting data returned from single surfaces, as well as multiple or distributed surfaces.

\section{Short-RAnge Time-OF-FLight PHOTON-COUNTING SYSTEM}

Our first laboratory-based experiments were performed in 1996 [2], and were primarily concerned with the demonstration of the basic principle. Although photon-counting time-of-flight had been used previously with retroreflective targets, most notably in satellite laser ranging [6], this demonstration attempted to establish whether the technique could be applied to high depth-resolution measurements over several meters, consistent with the application of noncontact reverse engineering of large mechanical parts.

Time-of-flight ranging using the TCSPC technique relies on the ability of measuring single-photon events with a timing accuracy of approximately the laser pulse duration. Critically, the timing accuracy can be improved by repeating the measurements many times (typically $10^{4}-10^{6}$ times) and averaging to achieve the necessary precision. The target is irradiated by a high 


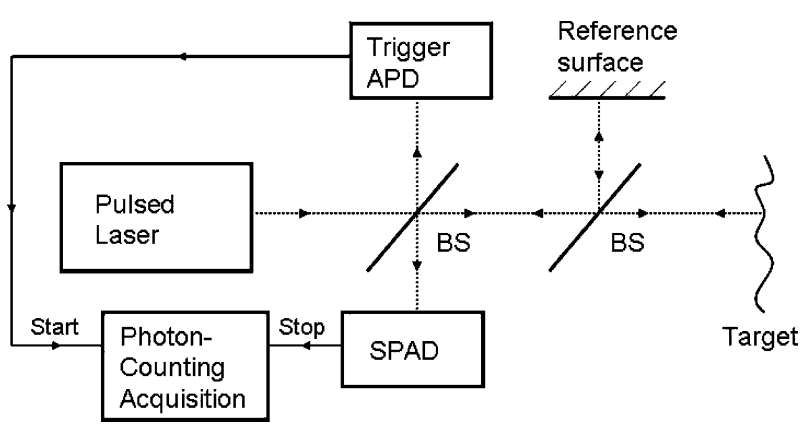

Fig. 1. Modular representation of photon-counting time-of-flight system. The dashed lines indicate optical paths while the solid lines indicate electrical connections. The "Start" and "Stop" channel for the timing measurement may be reversed depending on the type of acquisition hardware used. BS, optical beamsplitter; APD, avalanche photodiode.

repetition rate pulsed laser source, typically operating at a repetition frequency of $1-100 \mathrm{MHz}$, and the scattered return from the target is sensed by a single-photon detector. The sources used in these experiments were generally pulsed semiconductor lasers, typically outputting optical pulse energies of 1-100 pJ. The scattered return must be sufficiently attenuated so that the probability of a photon event is $<5 \%$ per pulse. Assuming that the measured data is normally distributed, if the error on a single timing measurement is $\sigma$, then the error, $x$, on the mean of $N$ measurements must be

$$
x=\frac{\sigma}{\sqrt{N}}
$$

Of course, such dependence means that even for $10^{6}$ counts, we can expect time resolution of the order of hundreds of femtoseconds for a 100-ps jitter TCSPC system, in the absence of systematic errors. In practice, we have previously demonstrated depth resolution of less than $30 \mu \mathrm{m}$ from such a system [7].

For reverse engineering of meter-dimensioned objects, a timeof-flight system based on TCSPC was developed. This sensor is based on the system shown schematically in Fig. 1. A pulsed semiconductor laser illuminates an object, with a small fraction of the beam directed to a linear multiplication avalanche photodiode to provide a start signal for the timing process. The remainder of the optical output is directed to the target and scattered returns are detected by the single-photon avalanche diode (SPAD) detector. The signal from these photon events is used to stop the timing process, and the output of the time differences between start and stop signals is displayed as a histogram. In practice, the system jitter is not the only possible source of error, and one major source can be drift, which manifests itself as a movement of the instrumental response along the time axis. Although the timescales of the drift tend to be relatively slow-greater than hundreds of milliseconds-this can affect measurements of $\sim 100$-ms duration or longer. To minimize the deleterious effects of drift on depth measurements, we introduced a fixed reference surface and then made the depth measurements between the return signals from each surface, as shown in Fig. 2. Any unpredictable drift during a measurement period means that both return signals are affected simultaneously, thus reducing the effect of this systematic error on the

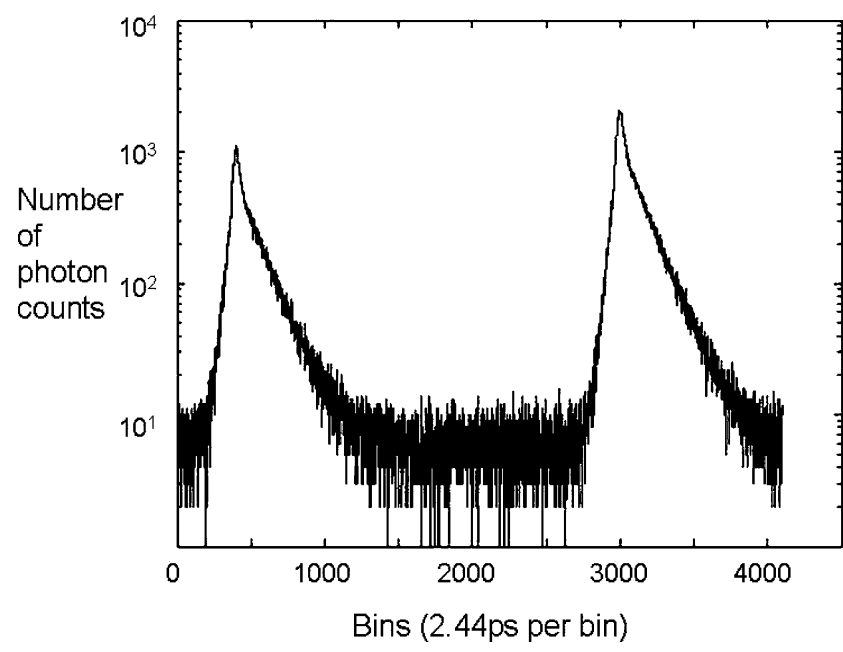

Fig. 2. Histogram of photon counts against bins using the short-range sensor described in Section II. In this example, 4096 bins of 2.44 ps per bin are illustrated. The histogram shows the target (left) and reference (right) response used to reduce the effects of drift in the timing system and provide an instrumental response.

depth measurement. In practice, the reference is delayed optically using a short length (a few meters) of fiber optical cable in order to bring the reference return into the same time window as the target return.

The implementation of this design is described in detail in [8], and is shown photographically in Fig. 3. The system was constructed using an anodized aluminum alloy baseplate, which was formed from a solid block of dimensions $260 \mathrm{~mm} \times 210 \mathrm{~mm} \times$ $100 \mathrm{~mm}$, with the optical axes formed by V-grooves cut using a computer-controlled milling machine. The pulsed laser used was a passively $Q$-switched AlGaAs laser diode [9] developed at the A.F. Ioffe Institute, St Petersburg, Russia. This laser emitted pulses at the 850-nm wavelength of between 10- and 20-ps duration and of pulse energy $7-10 \mathrm{pJ}$. The clean temporal pulse shape of these lasers complemented the use of the activelyquenched shallow junction Si SPAD detectors [10]-[12]. Such detectors are capable of 20-ps resolution timing at count rates in excess of $10^{6}$ per second. An advantage of these detectors in this application is their small active area $(\sim 7-\mu \mathrm{m}$ diameter $)$, which limited the detection of backreflections and ambient light by spatially filtering in the detector plane. The precise alignment is performed by placing each component within the accurately machined V-grooves and then fine-tuning using Risley prisms that are locked into position after the initial alignment procedure. Additional spectral filtering of ambient light is provided by optical bandpass filters. The design included a silicon chargecoupled device (CCD) camera for initial optical alignment. The collection lens used was a commercially available $200-\mathrm{mm}$ focal length camera lens (Pentax f/2.8). A Becker and Hickl SPC-300 data acquisition card was used with this short-range time-of-flight sensor. The depth repeatability of this sensor is described in more detail in Section IV.

To produce a scanned three-dimensional image, the entire system was placed on a pan-and-tilt head with angular repeatability of 5 arcsec. This is comparable to the optical spatial resolution 


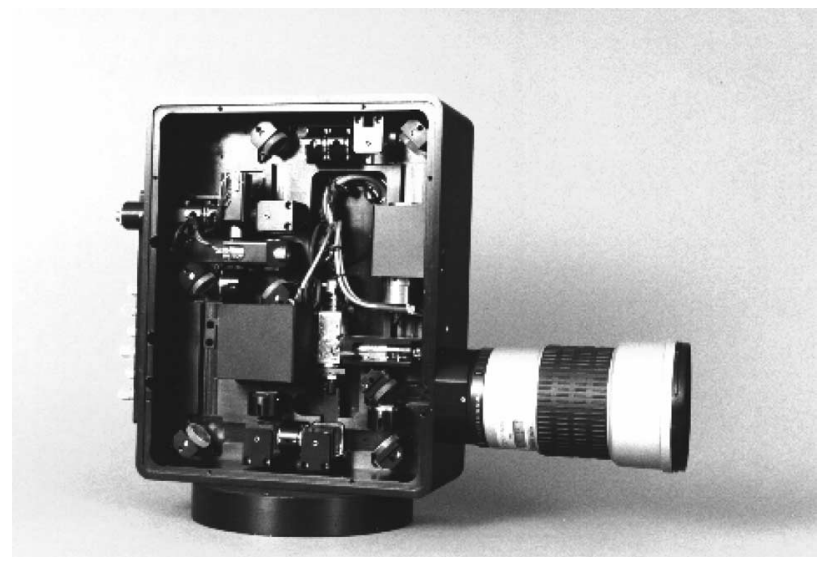

Fig. 3. Photograph of the photon-counting time-of-flight optical system. This assembly includes timing photodiode, single-photon avalanche diode, laser head, Si CCD camera, and all optics used for collimation, beamsplitting, alignment, and target return collection.

of the system, which is approximately $\sim 60 \mu \mathrm{m}$ at $2-\mathrm{m}$ standoff distance and $\sim 380 \mu \mathrm{m}$ at $13 \mathrm{~m}$, limited principally by the laser spot size of the system.

An example of a typical depth scan is shown in Fig. 4, illustrating a photograph and corresponding three-dimensional depth scan of an archaeological artefact. This artefact is a cast made in 1900 of a Roman frieze found in Scotland and dated second century A.D. In common with many delicate objects, historical artefacts of this nature cannot be subjected to a contact form of depth measurement, nor the use of potentially damaging high-power laser sources, making this method of depth profiling ideal for the purpose. Fig. 4(a) shows a photograph of the artefact and a depth profile of the frieze. The depth profile is a $307 \times 230$-pixel image with spatial resolution of $1 \mathrm{~mm}$ laterally and approximately $50 \mu \mathrm{m}$ in depth. More detailed discussion of data analysis approaches is given in Section IV.

\section{Multiple-WAVELENGTH Sensor FOR KilOMETER-RANGE DISTRIBUTED TARGETS}

The investigation of distributed targets-i.e., those containing more than one scattering surface - at greater than $1-\mathrm{km}$ range was investigated by use of a time-of-flight photon-counting system designed and constructed specifically for this purpose [13]. The system was also designed with multiple wavelengths in order to test the wavelength dependence of the distributed return from specific targets, in addition to examining other issues, such as atmospheric transmission, wavelength dependence of turbulence, and simultaneous acquisition of multiple photon-counting channels.

The optical system was based around a commercially available 200-mm diameter aperture Schmidt-Cassegrain telescope, and is shown schematically in Fig. 5 and described in more detail in [13]. In this system, a commercially available multiplepulsed laser diode system (PicoQuant Sepia) was employed. This laser system was composed of six individual pulsed laser diodes operating at wavelengths between 630 and $975 \mathrm{~nm}$. The laser diode heads, containing collimating optics, were placed
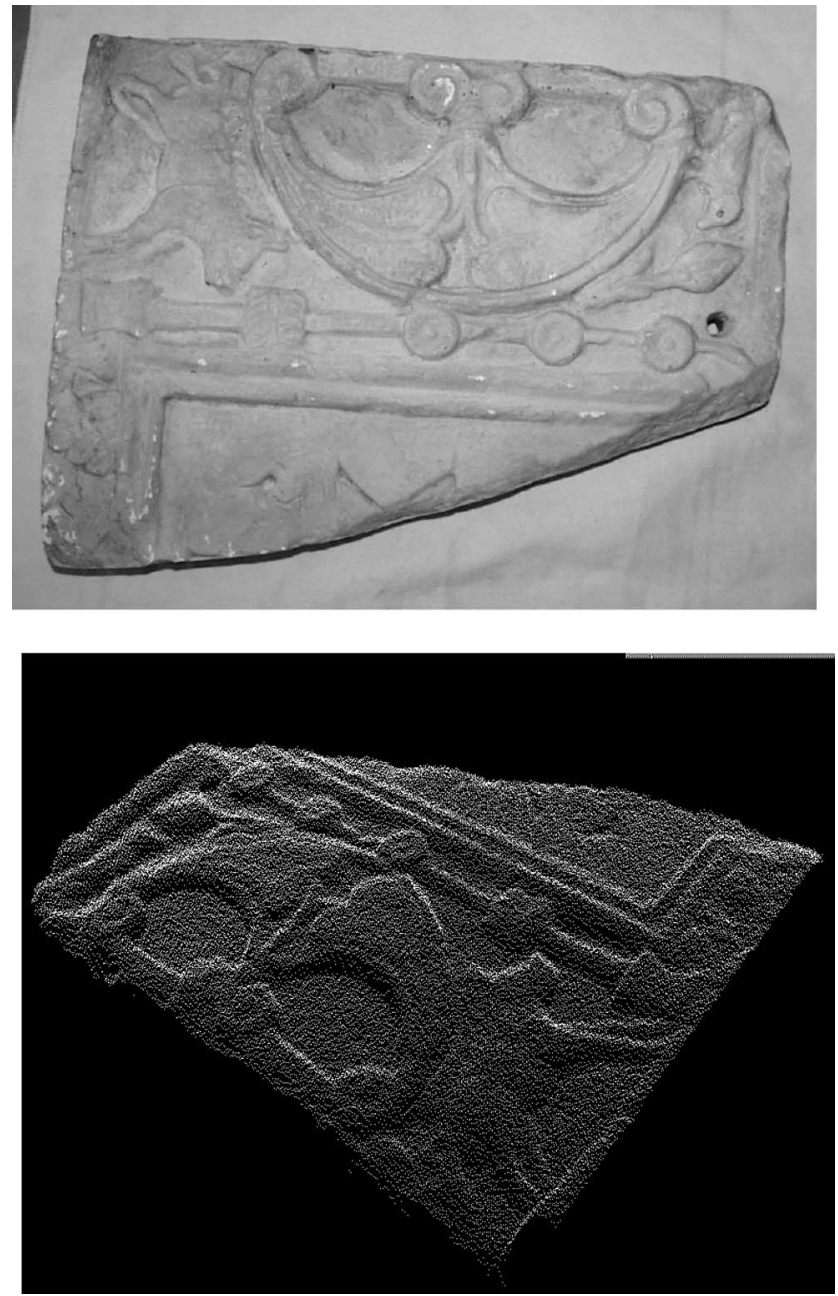

Fig. 4. Photograph of a Roman artefact (upper), and a point-cloud representation of a three-dimensional depth image of the same object (lower). The number of pixels in the depth image is $307 \times 230$ and the depth resolution is approximately $50 \mu \mathrm{m}$.

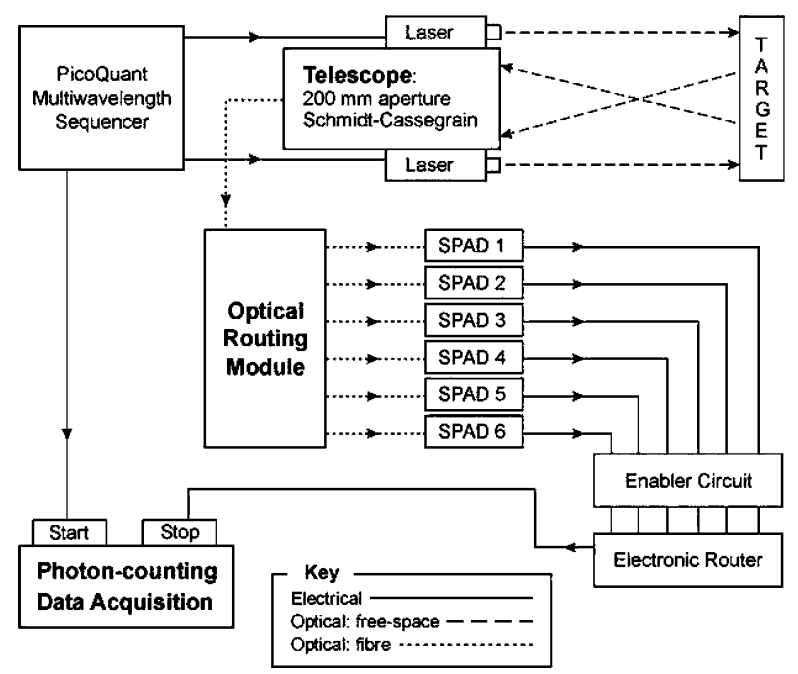

Fig. 5. Schematic representation of multiple-wavelength photon-counting sensor. The target returns from each of the six laser wavelengths are routed to each of the six SPADs. 
around the circumference of the telescope aperture and pulsed sequentially, so that only one laser would be operated at any given time, at an overall maximum repetition rate of $80 \mathrm{MHz}$. The collected returns were then coupled into an optical fiber and transferred to a custom-built optical routing module based on a plane-ruled diffraction grating, which multiplexed the six return wavelengths into six optical fibers connected to six individual SPAD modules (Perkin Elmer SPCM-AQR-12-FC). The optical routing module also provided spectral filtering for the solar background, which contributed the majority of background counts under normal operating conditions.

The six return signal inputs were fed to the photon-counting acquisition card (Becker and Hickl SPC-600) via an electronic router. However, the high background count rate resulting from the solar background under daylight operation meant that these spurious counts would result in a high proportion of dead time, resulting in inefficient signal collection. To counter this, an enabler circuit was developed, which sequentially allowed the output of each detector to be transferred to the data acquisition card, stopping all ambient dark counts outside the laser pulse window. This circuit consisted of a two-input NAND gate connected to each detector, with the other input connected to a synchronization signal derived from the laser driver. The laser synchronization used in the correct sequence meant that each detection channel passed only the counts expected in the time window, thus reducing all dark counts originating in other detectors.

A number of measurements were taken at ranges of up to $17 \mathrm{~km}$. An example is shown in Fig. 6. The response from a simple retroreflective target produces a quite different level of signal and background for each wavelength channel, but, in most cases, the signal is readily identified. This system was designed for analysis of scattered returns from distributed targets, and examples of this are shown in Section IV.

\section{INTERPRETING TIME-CORRELATED PHOTON-COUNT DATA}

In general, the performance of a light detection and ranging (LIDAR) system is a function not just of the optical design, the choice of detector, and the signal processing electronics, but also of the algorithms used to process the continuously acquired or stored data. For a TCSPC system, the data of interest is a discrete histogram of received photon counts in which each "bin" stores the accumulated returns for a given go-and-return transit time, directly proportional to the range to the target. In general, the integrated total of the received photon counts is not just dependent on the instrumental response, but also on the range and reflectance of the target, and the atmospheric transmission path. In tandem with the sensor and detector development, we have been investigating and developing a range of algorithms to process the TCSPC data to allow us to improve the detection rate and measurement accuracy of ranged surfaces, and better resolve closely separated returns, even when the surfaces are distant and of poor reflectivity. For a single pixel in a depth imaging system, or look direction in a spot ranging system, there may be several returns; this is because the beam may diverge

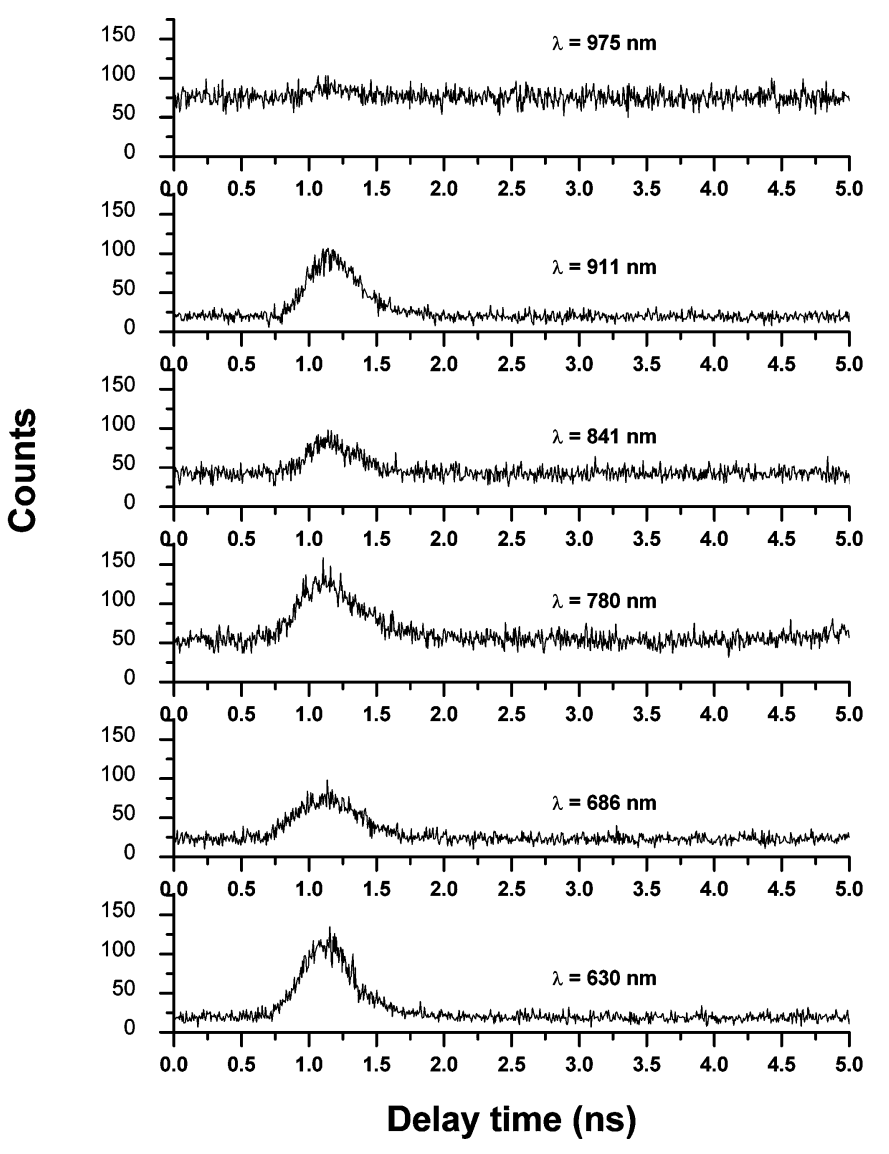

Fig. 6. Example of six simultaneous measurements for six different wavelengths from a corner cube target at $2-\mathrm{km}$ range.

and intersect with several surfaces, or, alternatively, be reflected from semitransparent surfaces in the same look direction.

In the simplest case, it is possible to obtain a range estimate by thresholding a prominent signal and reference peak in the TCSPC histogram, for example, at a fixed percentage of the peak height, and compute the temporal separation of the centroids of the thresholded data, and, hence, the distance to the target. An early example of the application of this approach was to determine the system depth precision from a number of repeated measurements of a single scattering surface placed at approximately $1 \mathrm{~m}$ from the sensor. An analysis of 20 measurements performed at six different levels of total integrated counts was made on the basis of this straightforward centroid methodand the difference in time (and, hence, the distance) between the target and reference was computed. The distance repeatability from these experimental measurements is shown in Fig. 7, and compared to those found from photon-counting data derived from a Monte Carlo simulation based on the experimentallydetermined instrumental response. The results clearly indicate the tradeoff between the number of integrated counts and the depth resolution as described in (1).

This approach has the advantage that it is nonparametric, i.e., it does not require knowledge of the instrumental response. If the instrumental response is known, then the results can be improved by previous matched filtering of the histogram with that response, improving the signal-to-noise ratio in an optimal 


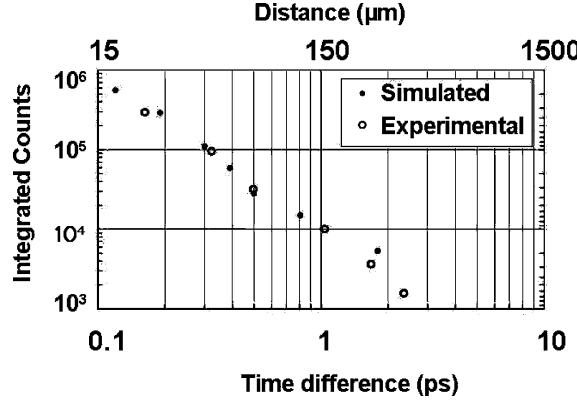

Fig. 7. Depth repeatability in terms of both time and distance as a function of integrated counts. Experimental results are shown in comparison to data derived from a Monte Carlo simulation. In both cases, the centroid method is used for data analysis.

fashion assuming that the Poisson noise can be approximated by a Gaussian function at high return levels.

However, this type of approach is not adequate for returns that are comparable to, or even below, the background level, since simple thresholding of the data fails to distinguish significant peaks. Further, if the histogram contains merged returns from closely separated surfaces, then this method fails to resolve the two peaks. The instrument used for a matched filter is no longer appropriate, and the centroid calculation is displaced to some point intermediate between the two real surface returns. For these reasons, we have developed more sophisticated approaches to process the TCSPC data.

Hence, assuming, in general, that there may be more than one return in a single pixel, the measured histogram, $F(i, \phi)$, is a statistical mixture from $k$ returns

$$
F(i, \phi)=\sum_{j=1}^{k} f_{j}\left(i, \phi_{j}\right)+B
$$

in which we use a set of four piecewise exponential (PE) functions to model the instrumental response of a single return

$$
f\left(i, \phi_{j}\right)=\beta \begin{cases}e^{\left(-\left(i_{1}-i_{0}\right)^{2} / 2 \sigma^{2}\right)} e^{\left(\left(i-i_{1}\right) / \tau_{1}\right)} & i \prec i_{1} \\ e^{-\left(i-i_{0}\right)^{2} / 2 \sigma^{2}} & i_{1} \leq i<i_{2} \\ e^{\left(-\left(i_{2}-i_{0}\right)^{2} / 2 \sigma^{2}\right)} e^{\left(-\left(i-i_{2}\right) / \tau_{2}\right)} & i_{2} \leq i \prec i_{3} \\ e^{\left(-\left(i_{2}-i_{0}\right)^{2} / 2 \sigma^{2}\right)} e^{\left(-\left(i_{3}-i_{2}\right) / \tau_{2}\right)} e^{\left(-\left(i-i_{3}\right) / \tau_{3}\right)} & i \geq i_{3} .\end{cases}
$$

This instrumental response is based on the characteristics of the SPAD detector, and was presented originally in [14]. The parameter $B$ represents the background photoncount level. The parameter set for a single return is $\phi_{j}=$ $\left\{\beta, \sigma, i_{0}, i_{1}, i_{2}, i_{3}, \tau_{1}, \tau_{2}, \tau_{3}\right\}_{j}$. In the methods that we have developed, we have generally kept this fixed when processing multiple returns, only allowing the parameter vector to vary for single surfaces. This is due to the complexity and the ill-posed nature of the problem. For example, if a response broadens, it is not determinable without prior knowledge whether this is due to a change in the parameter vector or to interaction of the beam with a surface distributed in space. Hence, the prior knowledge we would use is that the instrumental response is fixed. In the case of the metrology system, of course, we have a continuous instrumental return available from the fiber, so we can in theory change the parameter vector as we process the unknown data.

The number of photons, $c_{i}$, in bin $i$, is considered as a random sample from a Poisson distribution

$$
P\left(c_{i}\right)=e^{-F(i, \phi)} \frac{F(i, \phi)^{c_{i}}}{c_{i} !} .
$$

For a single histogram, we wish to determine the number of surface returns, the amplitude and time of arrival of each of these returns, and the return parameter vector, when allowed to vary from a reference (instrumental) response. In the next sections, we present increasingly complex and effective methods to achieve these goals.

\section{A. Scale-Space Filtering and Maximum Likelihood Estimation}

In the first approach, described fully in [15], we first apply a scale-space filtering [16], or bump hunting [17] procedure, that provides an initial estimate of the number, amplitude, and positions of the suspected returns. Depending on the amplitude and separation of discrete returns, a histogram of many returns may be multimodal or multitangential (implied by multimodality). Therefore, the TCSPC histogram is filtered by Gaussian derivative filters of progressively decreasing scale, i.e.,

$$
\begin{aligned}
G(i, h) & =\frac{1}{\sqrt{2 \pi} h} e^{-i^{2} / 2 h^{2}}, \quad G^{\prime}(i, h)=\frac{i}{\sqrt{2 \pi} h^{3}} e^{-i^{2} / 2 h^{2}} \\
G^{\prime \prime}(i, h) & =\frac{\left(i^{2} / h^{2}-1\right)}{\sqrt{2 \pi} h^{3}} e^{-i^{2} / 2 h^{2}}
\end{aligned}
$$

We then detect peaks in the first and second derivatives, rather than rely solely on zero-crossings in the first derivative [16]. This arises primarily from the desire to detect multiple returns when indicated only by multitangentiality, where zero crossings do not exist in the first-derivative filtered data. Fig. 8 illustrates this procedure on a simulated response that has four returns, the first three of which merge into a single mode on the left of Fig. 8(a). In the first derivative, shown in Fig. 8(b), smoothed with a kernel width of $h$ equal to 12 bins, there are zero-crossings corresponding to the modes at 235 and 500 bins. Combining an analysis of the first with the second derivative, we see that when the first derivative is positive, a negative peak in the second derivative shows an additional return (at 184 bins). When the first derivative is negative, a positive peak in the curvature shows an additional return (290 bins). Hence, in this example, the prediction of the scale-space filtering procedure is of four peaks at $184,235,290$, and 500 bins, respectively.

The scale-space filtering gives an ordered (by amplitude) list of possible peak positions and amplitudes. Next, we use an iterative procedure in which we add peaks from this list progressively and maximize the likelihood of the estimate of the parameters, $\phi$, in the mixture model

$$
L(c \mid \phi)=\prod_{i=1}^{N} \frac{F(i, \phi)^{c_{i}} e^{-F(i, \phi)}}{c_{i} !} .
$$

As it is not possible to have negative counts, and because the product term tends to zero, we minimize $-2 \ln L(c / \phi)$ as is the 

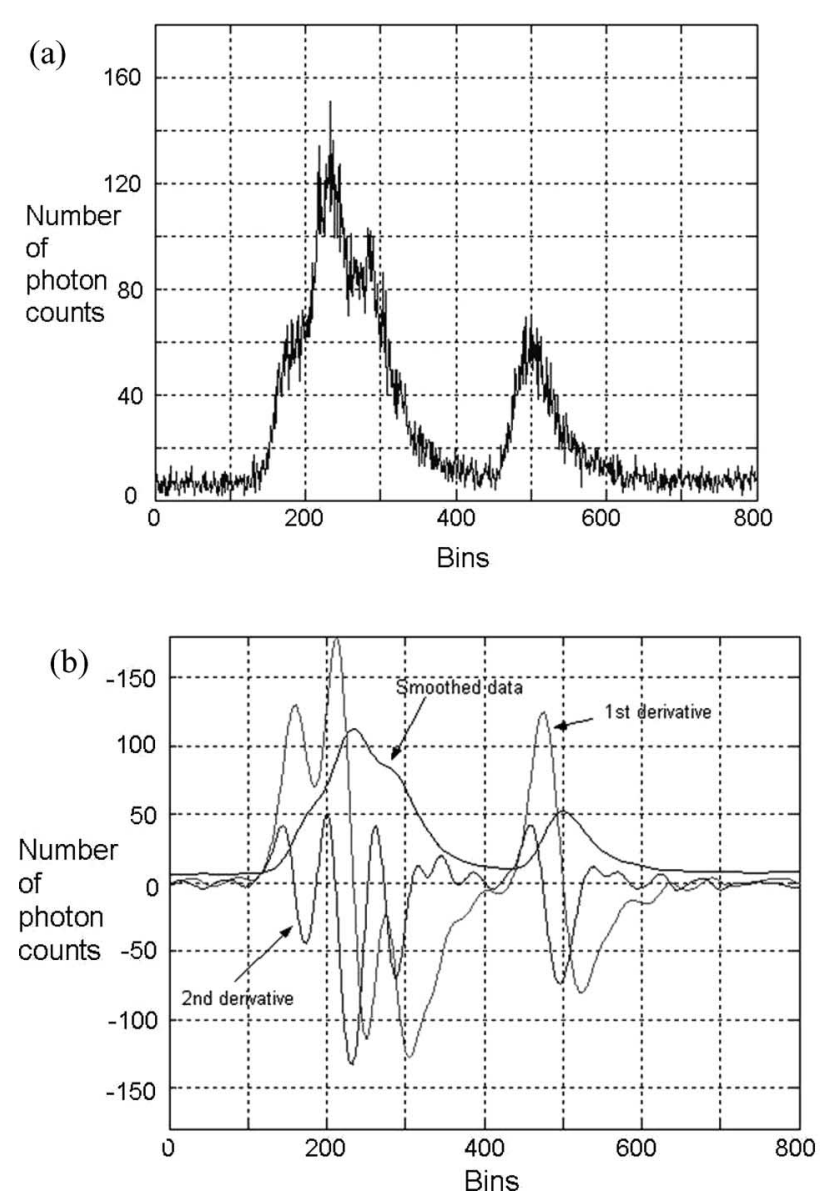

Fig. 8. (a) Simulation of four closely spaced returns with background, using Poisson statistics. (b) Smoothed data using a Gaussian of width 12 bins, and the first and second derivatives.

common practice [18]. The final term, $\ln \left(c_{i} !\right)$, is omitted as it is constant

$$
-2 \ln L(c \mid \phi)=2 \sum_{i=1}^{N}\left[F(i, \phi)-c_{i} \ln F(i, \phi)\right] .
$$

In general, there is a tendency to overfit the data, as adding more and more returns to fit the Poisson-distributed background can decrease the log-likelihood function. This should decrease rapidly, then remain approximately constant when the optimum interpretation of the number of returns is reached; at this point, we conclude the iterative process and return the final solution.

Considering Fig. 8, the final result from a maximum likelihood estimate is of four peaks of amplitudes 95.61(100), 69.65(45), 38.91(50), and 38.91(50), respectively (the true values are shown in parentheses). The positions were found to be 237.79(235), 284.86(290), 179.32(184), and 501.19(500), respectively. The background level was computed as 5.92(5) counts. Fig. 9 shows two details taken from full histograms taken from a single corner cube at a distance of $17 \mathrm{~km}$ in daylight conditions using the multispectral sensor described in Section III. In each case, the spectral channel was at $630 \mathrm{~nm}$ and the collection time was $100 \mathrm{~ms}$. The maximum likelihood estimate was of amplitude 31.09 counts at a position of 234.41 bins (a)
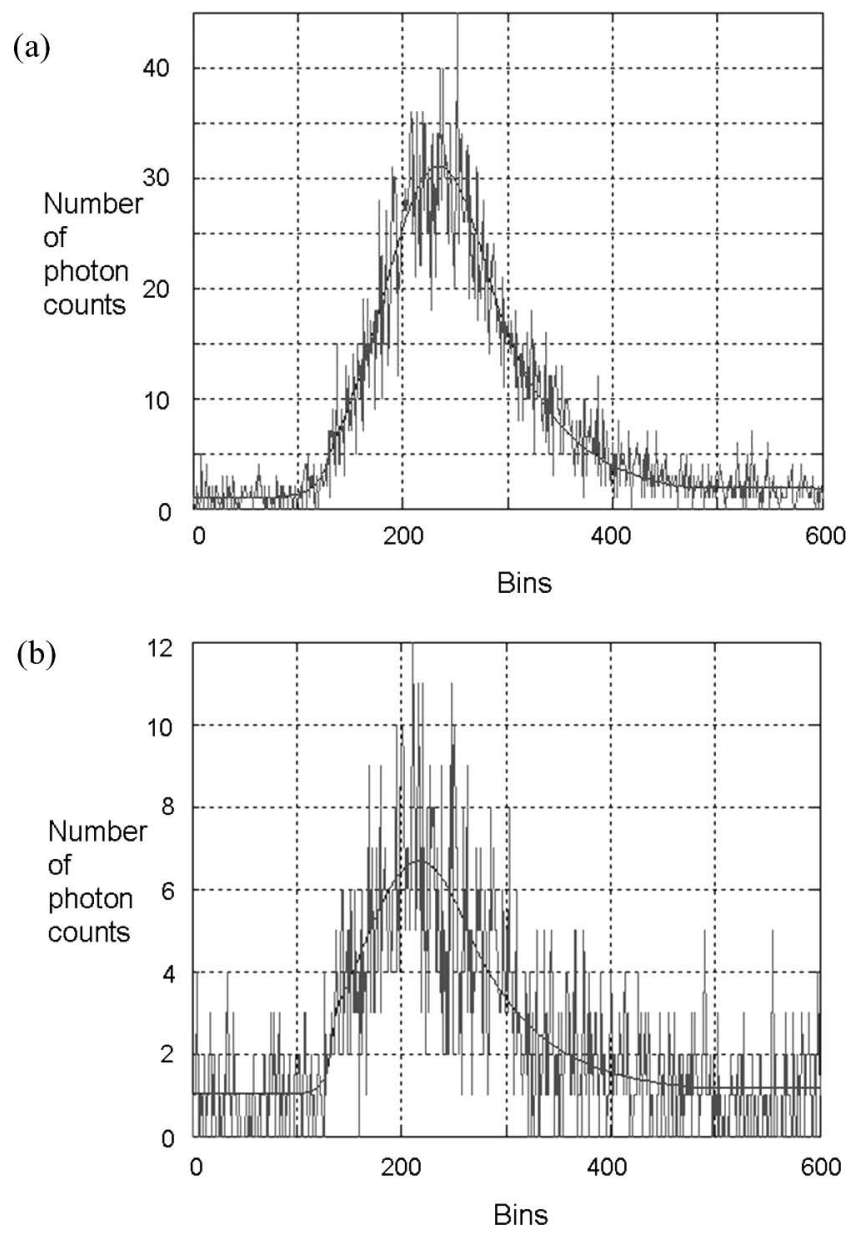

Fig. 9. Two examples of processed data from the multiple-wavelength sensor using the scale-space filtering (bump-hunting) and maximum likelihood estimation. (a) $841 \mathrm{~nm}$, collection time $1 \mathrm{~s}$. (b) $780 \mathrm{~nm}$, collection time $0.1 \sim \mathrm{s}$, both at a range of $17 \mathrm{~km}$.

with a background of 1.03 counts. In Fig. 9(b), the single return was of amplitude 6.71 counts at 217.07 bins, with a background of 1.03 counts.

\section{B. Reversible Jump Markov Chain Monte Carlo Processing (RJMCMC)}

Although the approach described in the previous section has been shown to be effective in many cases, this fails when the returns have amplitudes significantly lower than the background level, or if the returns are so closely separated that the estimation merges two returns into one. Therefore, we have progressed to apply MCMC and RJMCMC algorithms [19] to process TCSPC data, as reported in [20] and [21]. MCMC is a powerful simulation algorithm that allows us to find a stationary distribution, which is the posterior distribution of the parameters given the data (target distribution). This method is applicable to find the parameters for a known number of returns. However, RJMCMC techniques allow us to "jump" between different dimensional spaces, wherein our application of the dimension is determined by the number of returns. In one "sweep" of the RJMCMC algorithm, we have two steps. The first step is a parameter updating 

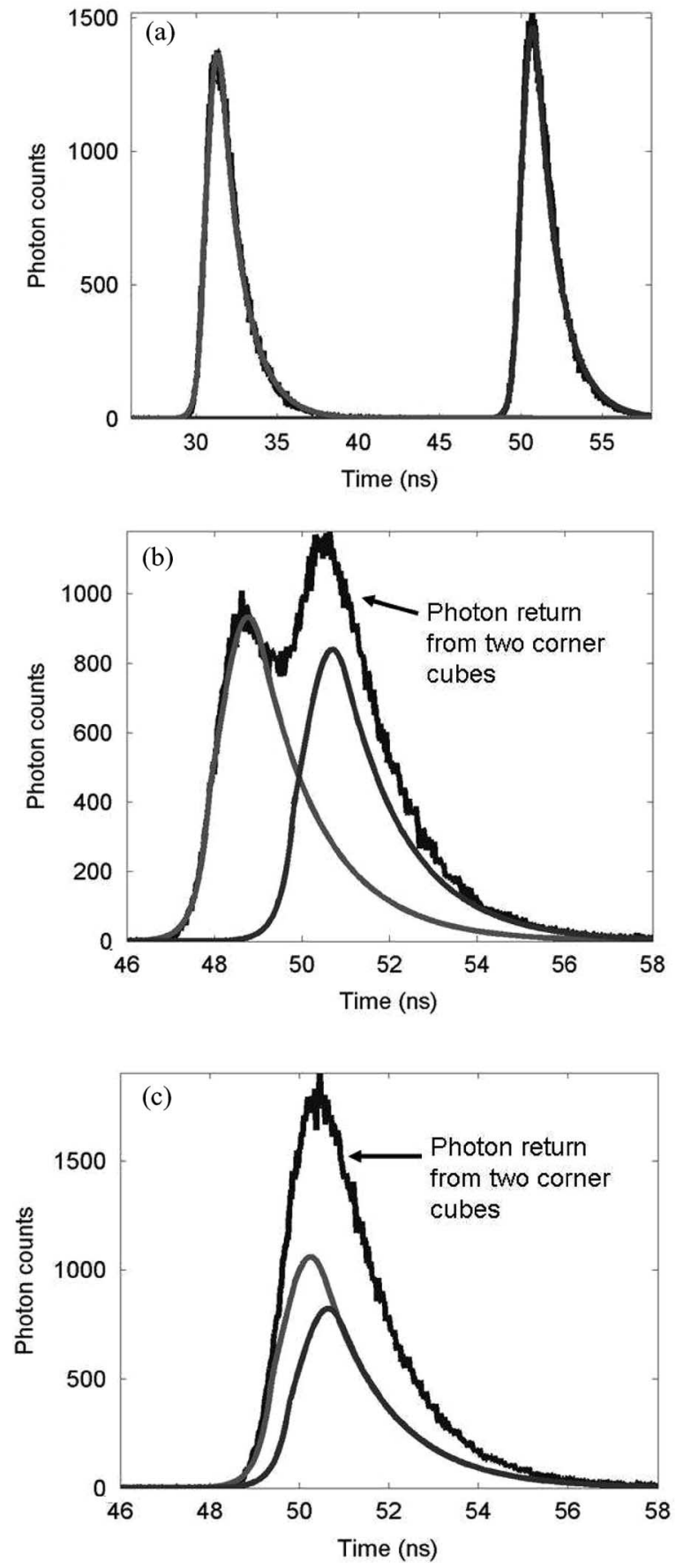

Fig. 10. Three examples of photon count distributions for two separated corner cubes at a standoff distance of $330 \mathrm{~m}$ at three different separations. Also shown are the solutions from the RJMCMC method, applied to find the number and separation of the returns. (a) The peaks are distinct and, hence, the actual return and the two predicted positions of the two returns are almost exactly overlaid. (c) Shows the return and calculated separation of the two returns for an actual corner cube separation of $1.7 \mathrm{~cm}$.

step, with fixed dimension, that improves the estimates of the set of amplitudes, time of arrival, and background parameters. The second is a dimension-changing step that allows jumps between spaces of different number of peaks. This can be either the birth of a new peak, the death of an existing peak, the splitting of

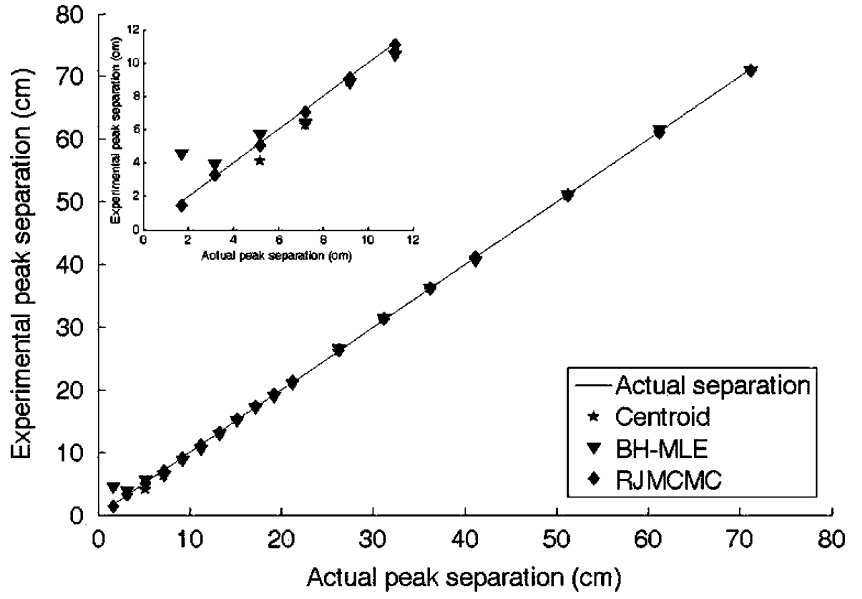

Fig. 11. A comparison of the three methods for measuring the separation of the corner cubes in comparison with ground truth (using the ruled gage).

an existing peak into two peaks, or the merging of two existing peaks into one peak.

A proposed move is accepted with the general probability

$$
\begin{aligned}
\alpha_{m}\left(k, \phi, k, \phi^{\prime}\right) \\
\quad=\min \left\{1, \frac{\pi\left(k^{\prime}, \phi^{\prime} \mid x\right)}{\pi(k, \phi \mid x)} \times \frac{q_{m}\left(k^{\prime}, \phi^{\prime}, k, \phi\right)}{q_{m}\left(k, \phi, k^{\prime}, \phi^{\prime}\right)}\right\}
\end{aligned}
$$

where $\alpha_{m}$ is the acceptance probability for a move from $(k, \phi)$ to $\left(k^{\prime}, \phi^{\prime}\right), k$ is the number of peaks, and $\phi$ is the parameter set for $k$ peaks. $\pi$ is the target distribution, $x$ the data distribution, $q_{m}$ the proposal distribution, and the subscript $m$ denotes the type of move. For parameter updating at a fixed number of returns, this reduces to the conventional Metropolis-Hastings probability

$$
\alpha\left(\phi, \phi^{\prime}\right)=\min \left\{1, \frac{\pi\left(\phi^{\prime} \mid x\right)}{\pi(\phi \mid x)} \times \frac{q_{m}\left(\phi^{\prime}, \phi\right)}{q_{m}\left(\phi, \phi^{\prime}\right)}\right\} .
$$

We have used a Gamma function for the proposed distribution of the amplitude and background parameters, dependent on the current values of $\beta$ and $B$, respectively. The proposed distribution for $i_{0}$ is a normal distribution that is also dependent on the current value. The birth/death and split/merge moves require a change of dimension and, therefore, RJMCMC is used. Considering the birth-death moves, the acceptance probability becomes

$$
\begin{aligned}
& \alpha_{m}\left(k, \phi, k, \phi^{\prime}\right) \\
& \quad=\min \left\{1, \frac{\pi\left(k^{\prime}, \phi^{\prime} \mid x\right)}{\pi(k, \phi \mid x)} \times \frac{r_{m}\left(\phi^{\prime}\right)}{r_{m}(\phi) q(u)} \times\left|\frac{\delta\left(\Phi^{\prime}\right)}{\delta(\Phi, u)}\right|\right\}
\end{aligned}
$$

where $r_{m}(x)$ is the probability of choosing move-type $m$ when in state $\phi, u$ is a vector of continuous random variables that ensures the reversibility of the deterministic function $\phi^{\prime}=h(\phi$, 

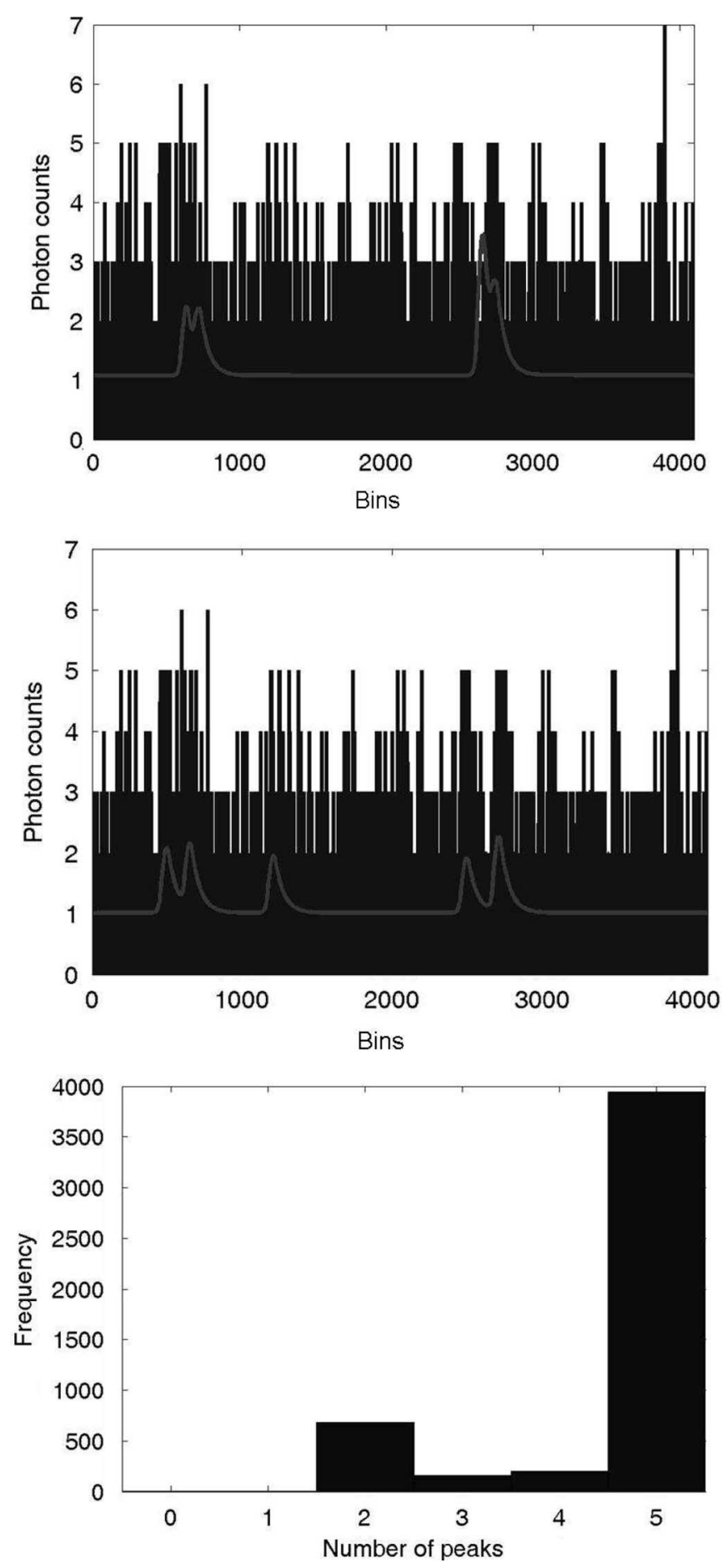

Fig. 12. A comparison of results for the BH-MLE (upper) and RJMCMC (middle) methods. The bottom graph shows the a posteriori distribution of the number of returns for the RJMCMC method, showing a clear probability of five peaks.

$u$ ), which allows the move to a higher-dimensional space and $q(u)$ is the probability density function of $u$. The Jacobian term, arising from the change of variable from $(\phi, u)$, to $\phi^{\prime}$, ensures the dimension balancing condition that allows reversible jumps between different dimensions of $k$. As in the previous section, the only a priori information that we assume is the instrumental
TABLE I

COMPARISON OF BH-MLE AND RJMCMC DATA ANALYSIS METHODS

\begin{tabular}{ccccccc}
\hline \hline & \multicolumn{2}{c}{ Actual values } & \multicolumn{2}{c}{ BH-MLE } & \multicolumn{2}{c}{ RJMCMC } \\
& Posn. & Ampl & Posn. & Ampl & Posn. & Ampl \\
\hline $1^{\text {st }}$ peak & 500 & 1 & 635.621 & 1.171 & 496.326 & 1.07 \\
$2^{\text {nd }}$ peak & 650 & 1 & 725 & 0.851 & 652.03 & 1.071 \\
$3^{\text {rd }}$ peak & 1200 & 1 & 2656 & 1.267 & 1207.367 & 0.944 \\
$4^{\text {th }}$ peak & 2500 & 1 & 2658 & 1.123 & 2494.939 & 0.909 \\
$5^{\text {th }}$ peak & 2700 & 1 & 2748 & 1.015 & 2707.642 & 1.239 \\
\hline \multirow{2}{*}{$\begin{array}{c}\text { Background } \\
\text { (B) }\end{array}$} & & 1 & & & & \\
\end{tabular}

response of the detector. The positions and amplitudes of the returned signals are drawn from appropriate prior distributions as explained in [20]. The number of peaks is usually set high to allow the algorithm to explore the whole space of solutions. A noninformative flat prior distribution is used for the number of peaks to reflect lack of knowledge about the number of returns.

We show results from experiments that demonstrate the improved performance of the RJMCMC method in comparison with the earlier bump hunting/scale-space filtering (BH-MLE) and centroid approaches. In Figs. 10 and 11 are shown the results from a series of trials in which two retroreflecting corner cubes were placed at a distance of $330 \mathrm{~m}$ from the multiplewavelength TCSPC sensor. The separation of these cubes was varied between 1.7 and $71.2 \mathrm{~cm}$ using a ruled gage. Applying each algorithm, we constrained the possible solutions to either zero, one, or two peaks. Fig. 10 shows the final stable measurements from the RJMCMC estimation at several distances; the algorithm is able to resolve the two surfaces even at the smallest resolution shown of $1.7 \mathrm{~cm}$, although this is not obvious to the human eye. Fig. 11 compares the RJMCMC approach to the BH-MLE and centroid methods. Whereas the centroid method was unable to determine the separation of peaks below $5.2 \mathrm{~cm}$, even with full a priori knowledge that two peaks were indeed present, both the BH-MLE and RJMCMC methods were able to do better than this. However, the RJMCMC method was able to determine the separation of peaks much more accurately at the smallest separation, seen clearly in the inset of Fig. 11 at an enlarged scale.

In addition to running a series of trials on real data, we have also performed systematic evaluation and comparison of our several algorithms using synthetic data. For these experiments, we generated a number of distributed (in position) returns of set amplitude and background level. The algorithms had no a priori knowledge of the numbers of returns or their parameters, except that a fixed instrumental response was used for (3). Fig. 12 shows the results of one such simulation and a comparison of the results from the BH-MLE and RJMCMC methods. Table I illustrates the true and estimated values for each of the methods. The distributions and the table show that, even when we do not have any a priori knowledge of the number of returns, the RJMCMC is able to infer the exact number of peaks, their 

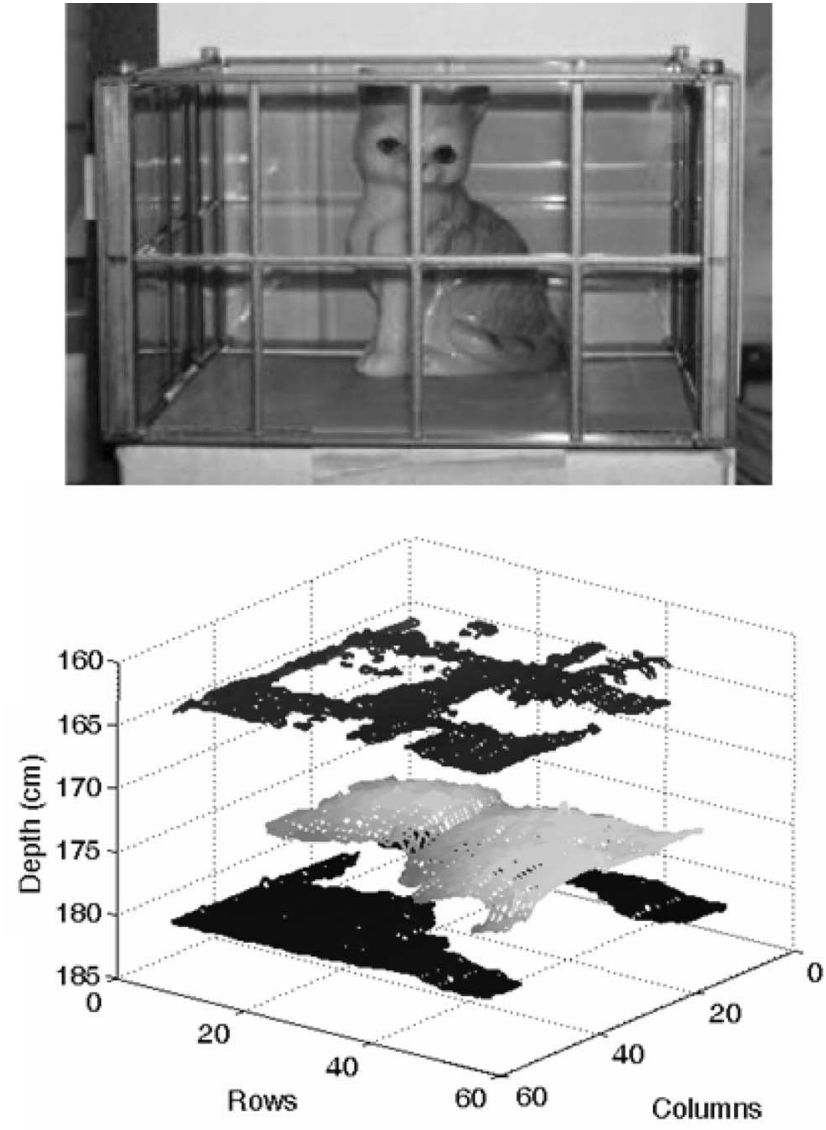

Fig. 13. A multilayered depth image of a cat figure inside a glass box. The top image shows a photograph of the cat, which has variable surface reflectivity, within the enclosure. The lower image shows that three distinct surfaces can be imaged, the front surface, the cat figure, and the surface behind the cat.

amplitudes, and their positions. In this case, it should be noted that the returns are very weak in comparison with the data of Fig. 10. In contrast, the BH-MLE algorithm fails to estimate the correct number and positions of peaks.

In Table I, to allow a comparison between the two methods, we have chosen to show the corresponding results for five peaks for the BH-MLE method, rather than using the log-likelihood function to set the threshold. Reading across the rows of the table, the much superior results from the RJMCMC method are evident. In the particular measurements used in this analysis, the system had been upgraded to include an adapted PerkinElmer detector, which had modifications to the single-photon detection circuitry [22] to improve jitter. With this modified detector and use of the RJMCMC algorithm, surface-to-surface depth resolution of $1.7 \mathrm{~cm}$ could be obtained at a distance of $330 \mathrm{~m}$ [23].

Fig. 13 illustrates an example of the application of the RJMCMC method to a full-depth image acquired by the sensor of Fig. 3, originally presented in [24]. In this case, there were reflections from the first surface of glass box, from a porcelain cat figure inside the box, and from the glass layer behind the cat. The consequent image is multilayered, although, in the majority of cases, there is a difficulty in detecting the first glass peak due to the predominantly specular nature of the reflection, i.e., the incident laser light was not scattered back toward the detector. In the middle portion of the cat, nearest normal incidence, both glass and cat surfaces are detected. Using this data, the distance between the glass surfaces was $18.0 \mathrm{~cm}$, consistent with the measured distance using a ruled gage.

\section{Conclusion}

This paper describes progress in time-of-flight ranging and imaging using TCSPC with point-by-point acquisition. The technique is fundamentally flexible: the tradeoff between the integrated number of counts (or acquisition time) against range repeatability or depth resolution allows its application in a number of diverse fields. The inherent time gating of the technique, allied to the spatial filtering provided by small active area, singlephoton detectors, can lead to operation under high ambient light conditions even with low average optical power sources.

We have demonstrated examples of three-dimensional imaging of meter-dimensioned objects where reverse engineering methods using cooperative targets cannot be routinely employed: e.g., mechanically delicate objects, or objects with more than one reflective surface. Using more advanced signal processing algorithms, we have been able to improve the system performance markedly, as measured by the depth resolution at shortand long range. Furthermore, the application of the BH-MLE and RJMCMC methodologies has allowed us to characterize the positions and amplitudes of multiple returns. Hence, the approach can be used for characterization of distributed noncooperative targets at kilometer ranges, even in environments where covert operation is necessary.

The technique of TCSPC in time-of-flight ranging and imaging applications is poised for more widespread application. However, to some extent, this will depend on further improvements to data acquisition hardware and detector performance, particularly in terms of jitter and detection efficiency. For some applications, however, it may result in more extensive infrared coverage.

\section{ACKNOWLEDGMENT}

The authors wish to thank their colleagues and collaborators for their valuable contributions and discussions over the past decade: R.A. Lamb, J.S. Massa, M. Umasuthan, S. HernandezMarin, G. Gibson, A. McCarthy, R.E. Warburton, S. Pellegrini, G.R. Smith, G.R. Mackinnon, R.C.W. Sung, R.D. Harkins, P.A. Hiskett, P. Czakany, C. Ayre, J.G. Rarity, C. Foster, and A.C. Walker. The passively $Q$-switched diodes were provided by Prof. Efim Portnoi and colleagues of the A.F. Ioffe Institute, St. Petersburg, Russia. The shallow-junction Si SPADs and modifications to the commercial Si SPAD module were provided by Prof. Sergio Cova and colleagues of the Politecnico di Milano, Italy. The cast of the Roman artefact was loaned by the National Museum of Scotland.

\section{REFERENCES}

[1] M. C. Amann, T. Bosch, M. Lescure, R. Myllylla, and M. Rioux, "Laser ranging: A critical review of usual techniques for distance measurement," Opt. Eng., vol. 40, no. 1, pp. 10-19, 2001. 
[2] J. S. Massa, A. M. Wallace, G. S. Buller, S. J. Fancey, and A. C. Walker, "Laser depth measurement based on time-correlated single-photon counting," Opt. Lett., vol. 22, no. 8, pp. 543-545, 1997.

[3] C. Ho, K. L. Albright, A. W. Bird, J. Bradley, D. E. Casperson, M. Hindman, W. C. Priedhorsky, W. R. Scarlett, R. C. Smith, J. Theiler, and S. K. Wilson, "Demonstration of literal three-dimensional imaging," Appl. Opt., vol. 38, no. 9, pp. 1833-1840, 1999.

[4] M. A. Albota, R. M. Heinrichs, D. G. Kocher, D. G. Fouche, B. E. Player, M. E. O'Brien, B. F. Aull, J. J. Zayhowski, J. Mooney, B. C. Willard, and R. R. Carlson, "Three-dimensional imaging laser radar with a photoncounting avalanche photodiode array and microchip laser," Appl. Opt, vol. 41, pp. 7671-7678, 2002.

[5] C. Niclass, A. Rochas, P.-A. Besse, and E. Charbon, "Design and characterization of a CMOS 3-D image sensor based on single photon avalanche diodes," IEEE J. Solid-State Circuits, vol. 40, no. 9, pp. 1847-1854, Sep. 2005.

[6] J. J. Degnan, "Thirty years of satellite laser ranging," presented at the 9th Int. Workshop Laser Ranging Instrum, Canberra, Australia, Nov. 1994.

[7] J. S. Massa, G. S. Buller, A. C. Walker, S. Cova, M. Umasuthan, and A. M. Wallace, "Time-of-flight optical ranging system using timecorrelated single photon counting," Appl. Opt., vol. 37, pp. 7268-7304, 1998.

[8] J. S. Massa, G. S. Buller, A. C. Walker, G. Smith, S. Cova, M. Umasuthan, and A. M. Wallace, "Optical design and evaluation of a 3-D imaging and ranging system based on time-correlated single photon counting," Appl. Opt., vol. 41, pp. 1063-1070, 2002.

[9] Z. I. Alferov, A. B. Zuralev, E. L. Portnoi, and N. M. Stel'makh, "Picosecond pulses from Q-switched heterostructure injection lasers," Sov. Phys. Tech., vol. 12, pp. 452-453, 1986.

[10] S. Cova, M. Ghioni, A. Lacaita, C. Samori, and F. Zappa, "Avalanche diodes and quenching circuits for single-photon detection," Appl. Opt., vol. 35, pp. 1954-1976, 1996.

[11] A. Lacaita, M. Ghioni, and S. Cova, "Double epitaxy improves singlephoton avalanche diode performance," Electron. Lett., vol. 25, pp. 841843, 1989.

[12] S. Cova, A. Lacaita, M. Ghioni, G. Ripamonti, and T. A. Louis, "20 ps timing resolution with single photon avalanche diodes," Rev. Sci.Instrum., vol. 60, pp. 1104-1110, 1989.

[13] G. S. Buller, R. D. Harkins, A. McCarthy, P. A. Hiskett, G. R. MacKinnon, G. R. Smith, R. Sung, A. M. Wallace, R. A. Lamb, K. D. Ridley, and J. G. Rarity, "A multiple wavelength time-of-flight sensor based on timecorrelated single-photon counting," Rev. Sci. Instrum., vol. 76, no. 8, pp. 083112-1-083112-7, 2005.

[14] S. Pellegrini, G. S. Buller, J. M. Smith, A. M. Wallace, and S. Cova, "Laser based distance measurement using picosecond resolution time-correlated single photon counting," J. Meas. Sci. Technol., vol. 11, pp. 712-716, 2000.

[15] A. M. Wallace, R. C. W. Sung, G. S. Buller, R. D. Harkins, R. E. Warburton, and R. A. Lamb, "Detecting and characterising return in a multi-spectral pulsed LADAR system," in Proc. IEE Vis. Image Signal Process., 2006, vol. 153 , no. 2 , pp. 160-172.

[16] P. Chaudhuri and J. S. Marron, "SiZer for exploration of structure in curves," J. Am. Stat. Assoc., vol. 94, no. 447, pp. 807-823, 1999.

[17] J. Harezlak, "Bump hunting in regression data revisited," M. Phil. thesis, Simon Fraser Univ., Burnaby, BC, Canada, 1998.

[18] M. D. Hannam and W. J. Thompson, "Estimating small signals by using maximum likelihood and Poisson statistics," Nucl. Instrum. Methods Phys. Res. A, Accel. Spectrom. Detect. Assoc. Equip., vol. 431, pp. 239$251,1999$.

[19] S. Richardson and P. J. Green, "On Bayesian analysis of mixtures with an unknown number of components," J. R. Stat. Soc. Ser. B, vol. 59, pp. 731-792, 1997.
[20] S. Hernandez-Marin, A. M. Wallace, and G. J. Gibson, "Bayesian analysis of LIDAR signals with multiple returns," IEEE Trans. Pattern Anal. Mach. Intell., to be published.

[21] S. Hernandez-Marin, A. M. Wallace, and G. J. Gibson, "Markov chain Monte Carlo algorithms for 3D ranging and imaging," presented at the IAPR Conf. Mach. Vis. Appl., Tsukuba, Japan, May 2005.

[22] I. Rech, I. LaBanca, M. Ghioni, and S. Cova, "Modified single photon counting modules for optimal timing performance," Rev. Sci. Instrum vol. 77, no. 3, pp. 033104-1-033104-5, 2006.

[23] R. E. Warburton, A. McCarthy, A. M. Wallace, S. Hernandez-Marin S. Cova, R. A. Lamb, and G. S. Buller, "Enhanced performance photoncounting time-of-flight sensor," Opt. Express, vol. 15, pp. 423-429, 2007.

[24] S. Hernandez-Marin, A. M. Wallace, and G. J. Gibson, "Creating multilayered 3D images using reversible jump MCMC algorithms," in Proc. Int Symp. Vis. Comput., Lect. Notes Comput. Sci., no. 4292, 2006, pp. 405416.

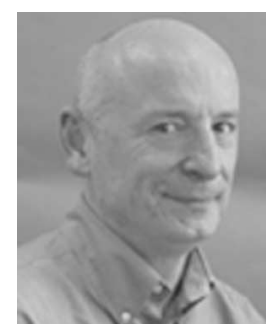

Gerald S. Buller (M'06) was born in Glasgow, Scotland, U.K., in 1965. He received the B.Sc. degree (with honors) in natural philosophy from the University of Glasgow, Glasgow, in 1986, and the $\mathrm{Ph} . \mathrm{D}$. degree in physics from the Department of Engineering and Physical Sciences, Heriot-Watt University, Riccarton, Edinburgh, U.K., in 1989, where he is currently a Professor of Physics.

In 2002, he founded Helia Photonics, Ltd., Livingston, Scotland. He is the author or coauthor of more than 200 papers published in intenational journals. He is the holder of several patents. His current research interests include aspects of single-photon detection, including infrared single-photon detectors, time-resolved photoluminescence, time-of-flight ranging, and quantum key distribution.

Prof. Buller is a Fellow of the Institute of Physics, U.K., and a member of the Optical Society of America.

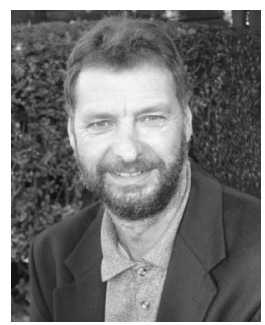

Andrew M. Wallace was born in St. Andrews, Scotland, U.K., in 1951. He received the B.Sc. degree in electrical engineering in 1972, and the Ph.D. degree in 1976, both from the University of Edinburgh, Edinburgh, Scotland.

Since 1984, he is with the Department of Engineering and Physical Sciences, Heriot-Watt University, Riccarton, Edinburgh. Currently, he is the Director of the Joint Research Institute in Signal and Image Processing at Heriot-Watt University, and the University of Edinburgh. He was a Research Fellow at the University of Glasgow, Glasgow, before joining Ferranti, Ltd., Oldham, Lancashire, U.K., as a Development Engineer in the Electro-Optics Group. He is the author or coauthor of several papers published in international journals. His current research interests include the development of laser systems for three-dimensional imaging, ranging, and classification.

Prof. Wallace is a Fellow of the Institution of Electrical Engineers, U.K., and the British Machine Vision Association. 